\title{
25 Research Square \\ Gut microbiome alterations predict diabetic kidney disease in general population
}

\section{Zhanzheng Zhao ( $\nabla$ zhanzhengzhao@zzu.edu.cn )}

Zhengzhou University First Affiliated Hospital https://orcid.org/0000-0001-6079-1631

\section{Weifeng Zhang}

Zhengzhou University First Affiliated Hospital

\section{Ruixue Guo}

Zhengzhou University First Affiliated Hospital

\section{Wen Cui}

Zhengzhou University First Affiliated Hospital

\section{Fanliang Zhang}

Zhengzhou University First Affiliated Hospital

\section{Yingyang Geng}

Zhengzhou University First Affiliated Hospital

\section{Chao Liu}

Institute of Biomedical Science

Jin Shang

Zhengzhou University First Affiliated Hospital

\section{Jing Xiao}

Zhengzhou University First Affiliated Hospital

\section{Xuejun Wen}

Virginia Commonwealth University

\section{Research article}

Keywords: Gut microbiome, diabetic kidney disease, community healthy individuals, non-invasive test

Posted Date: November 17th, 2020

DOI: https://doi.org/10.21203/rs.3.rs-104521/v1

License: (9) This work is licensed under a Creative Commons Attribution 4.0 International License. Read Full License 


\section{Abstract \\ Background}

Diabetic kidney disease (DKD) is increasingly prevalent owing to worldwide epidemic of diabetes mellitus (DM). The relationship between gut microbiome and chronic kidney disease (CKD) has attached wide attention during recent years. While the role of gut microbiome in diagnosis of DKD remains unclear.

\section{Methods}

We collected fecal samples of 180 patients with DKD and 179 healthy controls (Con) and characterized microbial profile using 16S rRNA gene sequencing approach. Microbial communities between the two groups were compared by Wilcoxon test. Two microbial models were identified by cross-validation preformed on random forest analysis. Diagnostic value of the two models was assessed in the training set including 118 DKDs and 132 healthy controls and validated in testing set composed of 62 DKDs and 47 healthy controls. PICRUSt was exploited to compare the potential metabolic function of microbiota between two groups.

\section{Results}

Compared with healthy controls, bacterial diversity in DKDs was decreased. Beta diversity showed markedly separating distribution between DKDs and Con groups. At the phylum level, DKD patients showed higher proportion of Proteobacteria, Verrucomicrobia and lower proportion of Bacteroidetes. In more details at the genus level, increase of opportunistic pathogens (including Escherichia-Shigella, Klebsiella, Enterococcus, Erysipelotrichaceae_incertae_sedis, Peptostreptococcaceae_incertae_sedis, Streptococcus) and mucin-degrading bacteria (including Akkermansia, Lactobacillus), as well as reduction of bacteria producing short-chain fatty acids (SCFA) such as Blautia[Faecalibacterium were also observed in DKDs. The optimal prediction model consisting of 5 OTUs could distinguish DKD from healthy controls with an area under the ROC curve of $83.71 \%$ in training set and $81.56 \%$ in testing set, while another 22 OTUs couldn't significantly improve diagnostic efficiency. Genomic metabolism associated with lipid and aromatic amino acids was over-expressed in DKD vs. Con.

\section{Conclusion}

Gut microbiome was altered in DKD patients. Our study suggested that gut microbiome may provide a potential tool for DKD diagnosis, especially those who could not accept or afford renal biopsy.

\section{Background}


Diabetic kidney disease (DKD), occurring in 30\%-40\% diabetes population, has become a public health problem worldwide due to the leading cause in end-stage renal disease (ESRD) [1]. As reported in 2015, DKD has surpassed glomerulonephritis to become the leading cause of chronic kidney disease (CKD) in Chinese hospitalized population ( $1.10 \%$ versus $0.75 \%$ )[2]. While, it is inaccurate to identify DKD only by appearance of micro-albuminuria or decreased glomerular filtration rate (GFR) in diabetic patients. It is reported that among the patients undergoing renal biopsy,DKD patients accounts for only $25 \%$ approximately. Additionally, laboratory indices lack effectiveness for early DKD diagnosis. Thus, novel test for early DKD identification is required.

Known as "endogenous microbial organ", commensal bacteria colonized in gut ferment indigestible carbohydrates, repair impaired intestinal barrier, competitively inhibit growth of "harmful" bacteria, participate in immune maintenance and access nutrients from host to drive host-microbe mutualism[3, 4]. Disruption of this stable ecosystem, characterized by increased potential pathogens or decreased "beneficial" bacteria, probably implies imbalance induced by disease. Accumulating researches have suggested that potential function of gut microbiome is closely related to fatty acid metabolism, oxidative stress resistance, regulation of insulin sensitivity in diabetes, as well as arteriosclerosis and thrombosis in atherosclerotic diseases[5-8]. Moreover, approach using microbial markers for diseases identification has been proposed and proven feasible. Diagnostic model consisting of genera Escherichia-Shigella and Prevotella_9 can distinguish 14 DNs from 14 DMs with 92\% accuracy[9]. Although the crucial role of gut microbiome in diseases development has been established within or beyond nephrology, microbial composition and function in DKD patients remains rarely reported. Therefore, a large cohort of $180 \mathrm{DKD}$ patients and 179 healthy controls are enrolled in our study to characterize gut microbiome, predict microbiome-associated function and develop a potential DKD classifier based on gut microbiome. Identification of structural and metabolic alteration of gut microbiome may help advance our understanding of gut-kidney axis.

\section{Methods And Materials}

\section{Patient selection}

We clustered quality-filtered sequences into unique sequences and identified abundance of representative sequences in a descending order using $U$ parse analysis. Sequences with $97 \%$ similarity to representative sequences were clustered and attributed to the same operational taxonomic units (OTUs). We finally obtained all OTUs of each sample from 180 patients with DKD and 179 healthy controls. The phylogenetic profile of each OTU was assigned against the Silva (SSU123)16S rRNA database by RDP Classifier (http://rdp.cme.msu.edu/) with a confidence threshold of $70 \%$. Data process of raw sequences was described in Supplementary file 1.

Depth of sequencing per sample was assessed by Rarefaction curve. Adequacy of sample size of DKD group and Con group was evaluated by species accumulation curve. Ace/Chao index and Shannon/Simpson index were used to calculate OTU richness and bacterial diversity respectively. Both 
principal coordinate analysis (PCoA) and non-metric multidimensional scaling (NMDS) plots were generated to visualize the (un)weighted UniFrac dissimilarity, corresponding statistical significance of which were tested using permutational multivariate analysis of variance (PERMANOVA) and analysis of similarities (ANOSIM) (QIIME package). The linear discriminant analysis (LDA) effect size (LEfSe) was applied to identify different microbial taxa and pathways (LDA score $>2.0$ and $\mathrm{P}$ value for Wilcoxon test $<0.5)$. Microbial metabolic pathways in both groups were identified using PICRUSt against Greengenes database.

\section{Statistical analysis}

Bacterial comparison from phylum to genus level between DKD and Con group were conducted using non-parametric Mann-Whitney $U$ test. To identify microbial markers with predictive potential, Wilcoxon test was used to calculate significance of the OTUs $(P<=0.05$ and $Q<=0.05)$, whose abundance in total was larger than 0.001 . After preliminary screening, parameters of importance were assigned to all potential OTU biomarkers by random forest. As a result, a number of 382 OTUs were identified as key linkage for cross-validation. With five trails of the five-fold cross-validation, the input features consisted of 5 OTUs were regarded as the optimal set with the minimum cv-error rate. Finally, the 5 OUTs' abundance profile of training set and testing set (as mentioned above) was used to calculate POD index for each sample. Area under the receiver operating characteristic (ROC) curve (AUC) was used to assess diagnostic effect. Similarly, microbial model consisted of 22 OTUs were identified as an alternative set when abundance index was defaulted as 0.005 . All statistical methods mentioned above were completed in $\mathrm{R}$ package.

\section{Results}

\section{A loss of microbial diversity in DKD}

Through the rigorous clinical inclusion and exclusion criteria, a total of 359 fecal samples were obtained for our study, including 179 healthy controls and 180 DKD patients. In agreement with Figure S1A, B, an average of 28349 reads per sample were collected in DKD group, lower than that 29348 of Con group. With sufficient sample size, number of detected OTUs showed a marked decrease in DKD compared with healthy controls (Figure S1C). For instance, there were total 2331 OTUs in DKD group, while 2997 in Con group (Figure 1A). Rank-abundance curve indicated a relatively uniform and diverse OTU profile in Con group (Figure S1D). By calculating index of alpha diversity, we observed a decrease of OTU richness (Ace for DKD vs. Con: 468.60 vs. $545.21 ; P=1.34$ e-05; Chao for $D K D$ vs. Con: 542.97 vs. $453.49 ; P=1.08 \mathrm{e}-06$ ) and diversity (Shannon for DKD vs. Con: 3.65 vs. 3.62; $P=5.19 \mathrm{e}-06$; Simpson for DKD vs. Con: 0.09 vs. $0.14 ; P=0.0048)$ in $D K D$ compared with Con (Figure 1B-E). To visualize spatial distribution of gut microbiome among all samples, (un)weighted UniFrac algorithm was used to compute beta diversity. PCoA analysis revealed significant difference between DKDs' and healthy controls' microbial community (PERMANOVA test for unweighted UniFrac distance: R2=0.0074, $P=0.001$; Figure 2F; PERMANOVA test for weighted UniFrac distance: $R 2=0.0036, P=0.197$, Figure $S 2 A$ ). Same results were also found in NMDS 
analysis based on Bray-Curtis distance (ANOSIM test: $\mathrm{R}=0.01342$, $\mathrm{P}=0.004$; Figure S2B) or unweighted UniFrac dissimilarity (ANOSIM test: $\mathrm{R}=0.02529 \otimes \mathrm{P}=0.001$; Figure $\mathrm{S} 2 \mathrm{C}$ ).

\section{Dysbacteriosis in gut of DKD}

At the phylum level, proportion of the four dominant bacteria (i.e., Firmicutes, Bacteroidetes, Proteobacteria and Actinobacteria) could achieve $95 \%$ of all sequences (Figure S3A). Compared with Con group, relative decrease of phyla Bacteroidetes and relative increase of Proteobacteria were observed in DKD, accompanying with increased Firmicutes/Bacteroidetes ratio (Figure 2A). At the class level, bacteria including Gammaproteobacteria, Verrucomicrobiae and Erysipelotrichia were abundant in DKD, while Betaproteobacteria and Bacteroidia were relatively decreased (Figure 2B). Correspondingly, a total of 16 bacteria at the order level and 31 bacteria at the family level were identified as significantly different between two groups (Figure 2C, 2D). In more detail at the genus level, we observed relative increase of potential pathogens consisting of Peptostreptococcus, Streptococcus, Peptostreptococcaceae_incertae_sedis, Erysipelotrichaceae_incertae_sedis, Enterococcus and Escherichia-Shigella, enrichment of sulphate-degrading bacteria Desulfovibrio, as well as decrease of "beneficial bacteria" including Roseburia, Blautia, Bacteroides and Faecalibacterium in DKD patients. Notably, relative abundance of Akkermansia, a mucin-degrading bacteria, was also reversely increased in DKD (Figure 2E, Figure S3B).

To evaluate microbial differences at the taxonomic level, LEfSe analysis was performed on microbial content of the 180 DKD patients and 179 healthy controls (LDA score $>2.0, P$ value $<0.05$ ). Besides those mentioned above, decreased abundance in genera including Lachnospira, Pseudobutyrivibrio and Anaerostipes and increased abundance in Bifidobacterium, Lactobacillus were observed in DKDs. We also demonstrated that genera Bacteroides (LDA=4.90, $P=1.95 \mathrm{e}-19)$ and Escherichia_Shigella (LDA=4.65区 $P=7.92 e-25 \rrbracket$ were strongly correlated with the disease status. All results above showed gut microbiome of DKD remarkably deviated from that of healthy status. (Figure S4).

\section{DKD identification based on OTUs}

Considering microbial striking difference in DKDs compared with healthy controls, a strategy based on potential microbiota-targeted markers for distinguishing DKD from healthy status was proposed. As mentioned in 'Statistical analysis' section, we performed random forest analysis on more than 1000 OTUs (number of sequences $>0.0001$ ) selected by Wilcoxon test $(P<0.05$ and $Q<0.05)$. As a result, 328 OTUs were identified as key markers and distribution of the first 73 OTUs (importance value $>0.001$ ) in 180 DKD patients and 179 healthy controls were shown in heatmap. Differential relative abundance between DKD and Con was observed (Figure 3). We also delineated the profile of top abundant 50 OTUs in each sample and found a relatively insignificant difference between DKD and Con group (Figure S5).

With five-fold cross validation performed on random forest model, an OTU combination consisted of 5 OTUs (model 1) was identified with the highest diagnostic value. As shown in Figure 4A, this optimal set had the minimum cv error-rate. These OTUs' contribution and stability in construction of disease classifier 
were respectively evaluated by decreasing accuracy and Gini (Figure 4B-C). Same identification method was further implemented on a range of OTUs whose abundance larger than 0.0005 and 22 OTUs were identified as another microbial combination with diagnostic value (Model 2; Figure 4D). Their function in model construction was shown in Figure 4E-F.

We applied ROC curve in the training set containing 118 DKD patients and 132 healthy controls and found combination of 5 OTUs could separate DKDs from healthy controls with $83.71 \%$ accuracy, while $81.56 \%$ in model 2 (Figure 5A). Although average POD values in DKD group were higher than Con group in both OTU sets, there was a more significant discrepancy in model 1 (Figure 5B, 5C). Remaining 62 DKDs and 47 healthy controls were incorporated into testing set to assess diagnostic efficiency of the two OTU sets. As expected, area under ROC curve (AUC) could achieve $80.89 \%$ in model 1 and $76.75 \%$ in model 2. Higher POD value in DKD than Con group was also observed in Figure 5D, 5E. These data suggested that increasing OTU markers couldn't improve predictive performance.

\section{Microbiome-associated functional changes in DKD}

PICRUSt was used to analyze microbiome-associated metabolism in disease or healthy status. A total of 145 KEGG pathways showed distinct relative abundance between DKD and Con group (LDA score $>2.0$ and $P$ value $<0.05$ ). Functional categories including membrane transportation ( $A B C$ transporters and phosphotransferase system), cell motility (bacterial motility proteins), lipid metabolism (fatty acid, glycerolipid, glycerophospholipid metabolism and unsaturated fatty acids biosynthesis), amino acid metabolism (aromatic amino acids, glutathione and others metabolism) and carbohydrate metabolism (pyruvate metabolism) showed higher levels in DKDs, while metabolism of cofactors, vitamins and specific amino acids (i.e., alanine, aspartate, glutamate, arginine and proline), oxidative phosphorylation were decreased in DKDs compared with healthy controls (Figure 6).

\section{Discussion}

In this study, we collected fecal samples of 180 DKD patients and 179 healthy individuals and used highthroughput 16S rRNA Miseq to complete the gut microbial profile mapping. Firstly, we elaborated on the obvious difference in diversity and abundance of gut microbes between DKD patients and healthy individuals. Compared with healthy individuals,the abundance and diversity of DKD patients' intestinal microbiota is relatively lower. Similar results were found in other kidney diseases such as chronic kidney disease and IgA nephropathy[10,11]. At the phylum level, the proportion of Proteobacteria and Verrucomicrobia are significantly increased, while Bacteroidetes decreased; at the genus level, the proportion of opportunistic pathogenic bacteria like Escherichia-Shigella, Klebsiella, Enterococcus, Erysipelotrichaceae_incertae_sedis, Peptostreptococcaceae_incertae_sedis, Peptostreptococcaceae_incertae $\llbracket$ were relatively increased. Secondly $\square$ by detecting changes in abundance of g_Lactobacillus, g_Sphingomonas, g_Gardnerella, g_Erysipelotrichace_Incertae_sedis,

g_Lachnospiraceae_unclassified $\square$ diabetic kidney disease can be accurately differentiated with the Health control group which has been matched with gender and age $(A U C=0.8089)$. In this study, compared with 
normal individuals, G Bacteroides and G Faecalibacterium were significantly decreased in DKD patients, whereas Escherichia-Shigella was significantly increased. Intriguingly, in Tao's study[12], genus Bacteroides was higher in DKD, while genus Bacteroides was significantly lower in DKD than in HC group in our study.

The metabolites of gut microbiota play pivotal roles in pathogenesis and health maintaining[13-15], especially SCFAs, ammonia, aromatic amino acids, butyric acid, indole and p-cresol[16, 17]. The single layer of epithelial cells that makes up the mucosal interface between the host and microorganisms allows microbial metabolites to gain access to and interact with host cells, and thus influence immune responses and disease risk[15]. Compared with HC group, DKD group's metabolism of amino acids, particularly glutamine, were significantly decreased. A study reported amino acids level in intestine was lower, which may be due to the large amount of nitrogen resources is needed to maintain protein synthesis during gut microbiota proliferation or intestinal tissues growth[16, 18]. It is reported that intestinal microbes have critical role in the normal development of organized lymphoid structures and in the regulation of host immune function[15, 19-21]. It has recently become evident that individual commensal species influence the makeup of lamina propria $T$ lymphocyte subsets that have distinct effector functions [15].

The study by Jiang $S$ et al [22] reported that Coprococus and Faecalibacterium were significantly lower in CKD than the normal group. Similarly, compared with HC group, they were significantly lower in DKD group. Coprococcus and Faecalibacterium belong to Firmicutes-Clostridiales and can produce butyrate. Butyrate regulates $\mathrm{T}$ cells by up-regulating $\mathrm{H} 3$ and $\mathrm{H} 4$ histone acetylation of Fox 3 site, which in turn affects the process of systemic inflammation and diabetic kidney disease. Butyrate production is influenced by diet[23], but study by Tao $S$ et al [12]showed gut microbiota composition of Household $(\mathrm{HH})$ and Healthy Control groups were similar, but significantly different with DN group under identical diet, which indicates that diseases play an important role in promoting gut microbiota composition alterations. However, study by Poesen.R et al. [24]found that the gut microbiota composition of Household group and the CKD group, which have the same diet, gender, age and BMI, were similar.

This study only included DKD patients without other comorbid diseases, while in real clinical practice, most of DKD patients combine with multiple chronic diseases (e.g. cardiovascular diseases, tumors, Alzheimer's disease, CKD etc.) which may also influence abundance and diversity of gut microbiota[7, 25-28]. The correlation between gut microbiota composition and lifestyle or specific medications need to be further clarified at the same time. Therefore,further studies on determining characteristic intestinal microbes markers for DKD diagnosis are required.

\section{Conclusion}

This study found structural and metabolic alterations of DKD patients' gut microbiota. Based on this, the study may provide a potential non-invasive diagnostic method for DKD by identifying the unique gut 
microbiota composition. It may also be the option of early screening and prediction for DKD in community healthy individuals.

\section{Abbreviations}

\begin{tabular}{|ll|}
\hline DKD & diabetic kidney disease \\
\hline Con group & control group \\
DM & diabetes mellitus \\
CKD & chronic kidney disease \\
SCFA & short-chain fatty acids \\
\hline PICRUSt & Phylogenetic Investigation of Communities by Reconstruction of Unobserved States \\
\hline ESRD & end-stage renal disease \\
\hline OTUs & operational taxonomic units \\
\hline PCoA & principal coordinate analysis \\
\hline NMDS & non-metric multidimensional scaling \\
\hline PERMANOVA & permutational multivariate analysis of variance \\
\hline ANOSIM & analysis of similarities \\
\hline LDA & linear discriminant analysis \\
\hline LEfSe & linear discriminant analysis effect size \\
\hline POD & probability of disease \\
\hline ROC & receiver operating characteristic \\
\hline AUC & area under the receiver operating characteristic curve \\
\hline
\end{tabular}

\section{Declarations}

\section{Acknowledgements}

Not applicable.

\section{Authors' contributions}

ZZZ and JS designed the study; WZ carried out experiments; WZ, RG, WC, FZ and YG collected samples; JS and CL analyzed the data and made the figures; JX, XW and JS drafted and revised the paper. All authors approved the final version of the manuscript. 


\section{Funding}

This work was supported by the National Natural Science Foundation of China (Grant Nos. 81873611 and 81700633). The funders designed the study and approved publication of manuscript.

\section{Availability of data and materials}

The datasets used and/or analyzed during the current study are available from the corresponding author on reasonable request.

\section{Ethics approval and consent to participate}

The present study was conducted in accordance with the Declaration of Helsinki and was approved by the Institutional Review Board of the first affiliated hospital of Zhengzhou university, school of medicine(2019-KY-361). All patients provided written informed consent.

\section{Consent for publication}

Written informed consent from the reported patient for publication was obtained.

\section{Competing interests}

The authors declare that they have no competing interests.

\section{Footnotes}

Publisher's Note

Springer Nature remains neutral with regard to jurisdictional claims in published maps and institutional affiliations.

\section{Contributor Information}

Weifeng Zhang, Email: zwf1520739@163.com

Ruixue Guo, Email: grx19960321@163.com

Wen Cui, Email: 498522802@qq.com

Fanliang Zhang, Email: 276553615@qq.com

Yingyang Geng, Email: 2020943995@qq.com

Chao Liu, Email: chaoliu@mobiomed.com

Jin Shang, Email: fccshangj2@zzu.edu.cn 
Jing Xiao, Email: xiaojing5123@139.com

Xuejun Wen, Email: xjwen@clemson.edu

Zhanzheng Zhao, Email: zhanzhengzhao@zzu.edu.cn

\section{References}

1. Umanath K, Lewis JB: Update on Diabetic Nephropathy: Core Curriculum 2018. Am J Kidney Dis 2018, 71(6):884-895.

2. Zhang L, Long J, Jiang W, Shi Y, He X, Zhou Z, Li Y, Yeung RO, Wang J, Matsushita K et al: Trends in Chronic Kidney Disease in China. N Engl J Med 2016, 375(9):905-906.

3. Liang Q, Chiu J, Chen Y, Huang Y, Higashimori A, Fang J, Brim H, Ashktorab H, Ng SC, Ng SSM et al: Fecal Bacteria Act as Novel Biomarkers for Noninvasive Diagnosis of Colorectal Cancer. Clin Cancer Res 2017, 23(8):2061-2070.

4. Baxter NT, Ruffin MTt, Rogers MA, Schloss PD: Microbiota-based model improves the sensitivity of fecal immunochemical test for detecting colonic lesions. Genome Med 2016, 8(1):37.

5. Brunkwall L, Orho-Melander M: The gut microbiome as a target for prevention and treatment of hyperglycaemia in type 2 diabetes: from current human evidence to future possibilities. Diabetologia 2017, 60(6):943-951.

6. Tuttle KR, Bakris GL, Bilous RW, Chiang JL, de Boer IH, Goldstein-Fuchs J, Hirsch IB, Kalantar-Zadeh K, Narva AS, Navaneethan SD et al: Diabetic kidney disease: a report from an ADA Consensus Conference. Am J Kidney Dis 2014, 64(4):510-533.

7. Peng W, Yi P, Yang J, Xu P, Wang Y, Zhang Z, Huang S, Wang Z, Zhang C: Association of gut microbiota composition and function with a senescence-accelerated mouse model of Alzheimer's Disease using 16S rRNA gene and metagenomic sequencing analysis. Aging (Albany NY) 2018, 10(12):4054-4065.

8. Zhang B, Sun W, Yu N, Sun J, Yu X, Li X, Xing Y, Yan D, Ding Q, Xiu Z et al: Anti-diabetic effect of baicalein is associated with the modulation of gut microbiota in streptozotocin and high-fat-diet induced diabetic rats. Journal of Functional Foods 2018, 46:256-267.

9. Wang L, Li P, Tang Z, Yan X, Feng B: Structural modulation of the gut microbiota and the relationship with body weight: compared evaluation of liraglutide and saxagliptin treatment. Sci Rep 2016, 6:33251.

10. Sabatino A, Regolisti G, Brusasco I, Cabassi A, Morabito S, Fiaccadori E: Alterations of intestinal barrier and microbiota in chronic kidney disease. Nephrol Dial Transplant 2015, 30(6):924-933.

11. De Angelis M, Montemurno E, Piccolo M, Vannini L, Lauriero G, Maranzano V, Gozzi G, Serrazanetti D, Dalfino $G$, Gobbetti $M$ et al: Microbiota and metabolome associated with immunoglobulin $A$ nephropathy (IgAN). PLoS One 2014, 9(6):e99006. 
12. Tao S, Li L, Li L, Liu Y, Ren Q, Shi M, Liu J, Jiang J, Ma H, Huang Z et al: Understanding the gut-kidney axis among biopsy-proven diabetic nephropathy, type 2 diabetes mellitus and healthy controls: an analysis of the gut microbiota composition. Acta Diabeto/ 2019, 56(5):581-592.

13. Hobby GP, Karaduta O, Dusio GF, Singh M, Zybailov BL, Arthur JM: Chronic kidney disease and the gut microbiome. Am J Physiol Renal Physiol 2019, 316(6):F1211-f1217.

14. Rooks MG, Garrett WS: Gut microbiota, metabolites and host immunity. Nat Rev Immunol 2016, 16(6):341-352.

15. Sekirov I, Russell SL, Antunes LC, Finlay BB: Gut microbiota in health and disease. Physiol Rev 2010, 90(3):859-904.

16. Kieffer DA, Piccolo BD, Vaziri ND, Liu S, Lau WL, Khazaeli M, Nazertehrani S, Moore ME, Marco ML, Martin RJ et al: Resistant starch alters gut microbiome and metabolomic profiles concurrent with amelioration of chronic kidney disease in rats. Am J Physiol Renal Physio/ 2016, 310(9):F857-871.

17. Wong J, Piceno YM, DeSantis TZ, Pahl M, Andersen GL, Vaziri ND: Expansion of urease- and uricasecontaining, indole- and p-cresol-forming and contraction of short-chain fatty acid-producing intestinal microbiota in ESRD. Am J Nephrol 2014, 39(3):230-237.

18. Mardinoglu A, Shoaie S, Bergentall M, Ghaffari P, Zhang C, Larsson E, Bäckhed F, Nielsen J: The gut microbiota modulates host amino acid and glutathione metabolism in mice. Mol Syst Biol 2015, 11(10):834.

19. Hooper LV, Littman DR, Macpherson AJ: Interactions between the microbiota and the immune system. Science 2012, 336(6086):1268-1273.

20. Atarashi K, Tanoue T, Shima T, Imaoka A, Kuwahara T, Momose Y, Cheng G, Yamasaki S, Saito T, Ohba $Y$ et al: Induction of colonic regulatory T cells by indigenous Clostridium species. Science 2011, 331(6015):337-341.

21. Round JL, Mazmanian SK: Inducible Foxp3+ regulatory T-cell development by a commensal bacterium of the intestinal microbiota. Proc Natl Acad Sci U S A 2010, 107(27):12204-12209.

22. Jiang S, Xie S, Lv D, Wang P, He H, Zhang T, Zhou Y, Lin Q, Zhou H, Jiang J et al: Alteration of the gut microbiota in Chinese population with chronic kidney disease. Sci Rep 2017, 7(1):2870.

23. David LA, Maurice CF, Carmody RN, Gootenberg DB, Button JE, Wolfe BE, Ling AV, Devlin AS, Varma Y, Fischbach MA et al: Diet rapidly and reproducibly alters the human gut microbiome. Nature 2014, 505(7484):559-563.

24. Poesen R, Windey K, Neven E, Kuypers D, De Preter V, Augustijns P, D'Haese P, Evenepoel P, Verbeke K, Meijers B: The Influence of CKD on Colonic Microbial Metabolism. J Am Soc Nephrol 2016, 27(5):1389-1399.

25. Ren Z, Li A, Jiang J, Zhou L, Yu Z, Lu H, Xie H, Chen X, Shao L, Zhang R et al: Gut microbiome analysis as a tool towards targeted non-invasive biomarkers for early hepatocellular carcinoma. Gut 2019, 68(6):1014-1023.

26. Liu H, Chen X, Hu X, Niu H, Tian R, Wang H, Pang H, Jiang L, Qiu B, Chen X et al: Alterations in the gut microbiome and metabolism with coronary artery disease severity. Microbiome 2019, 7(1):68. 
27. Li Y, Su X, Zhang L, Liu Y, Shi M, Lv C, Gao Y, Xu D, Wang Z: Dysbiosis of the gut microbiome is associated with CKD5 and correlated with clinical indices of the disease: a case-controlled study. $J$ Transl Med 2019, 17(1):228.

28. Sabatino A, Regolisti G, Cosola C, Gesualdo L, Fiaccadori E: Intestinal Microbiota in Type 2 Diabetes and Chronic Kidney Disease. Curr Diab Rep 2017, 17(3):16.

\section{Figures}

A

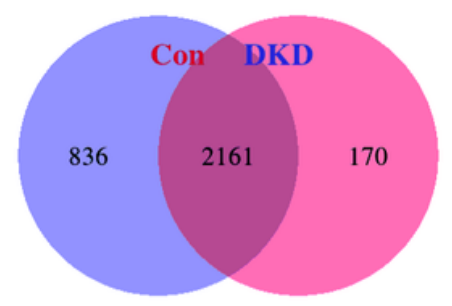

B

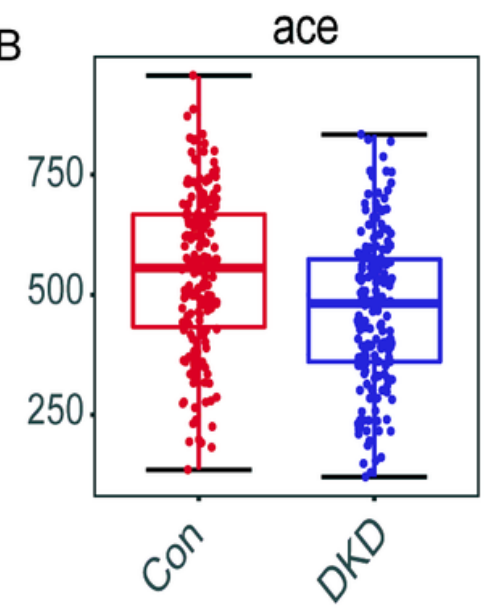

C

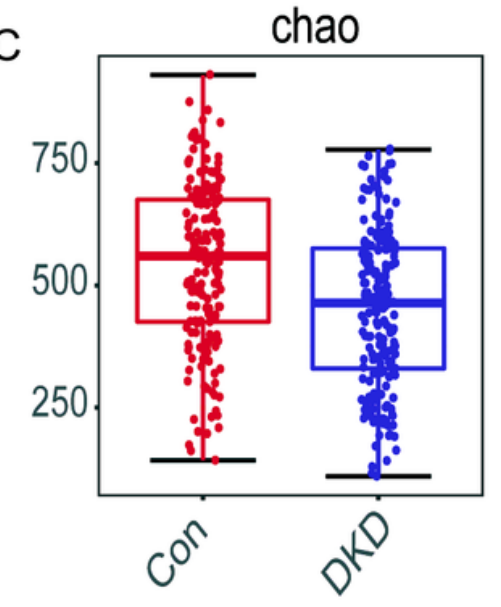

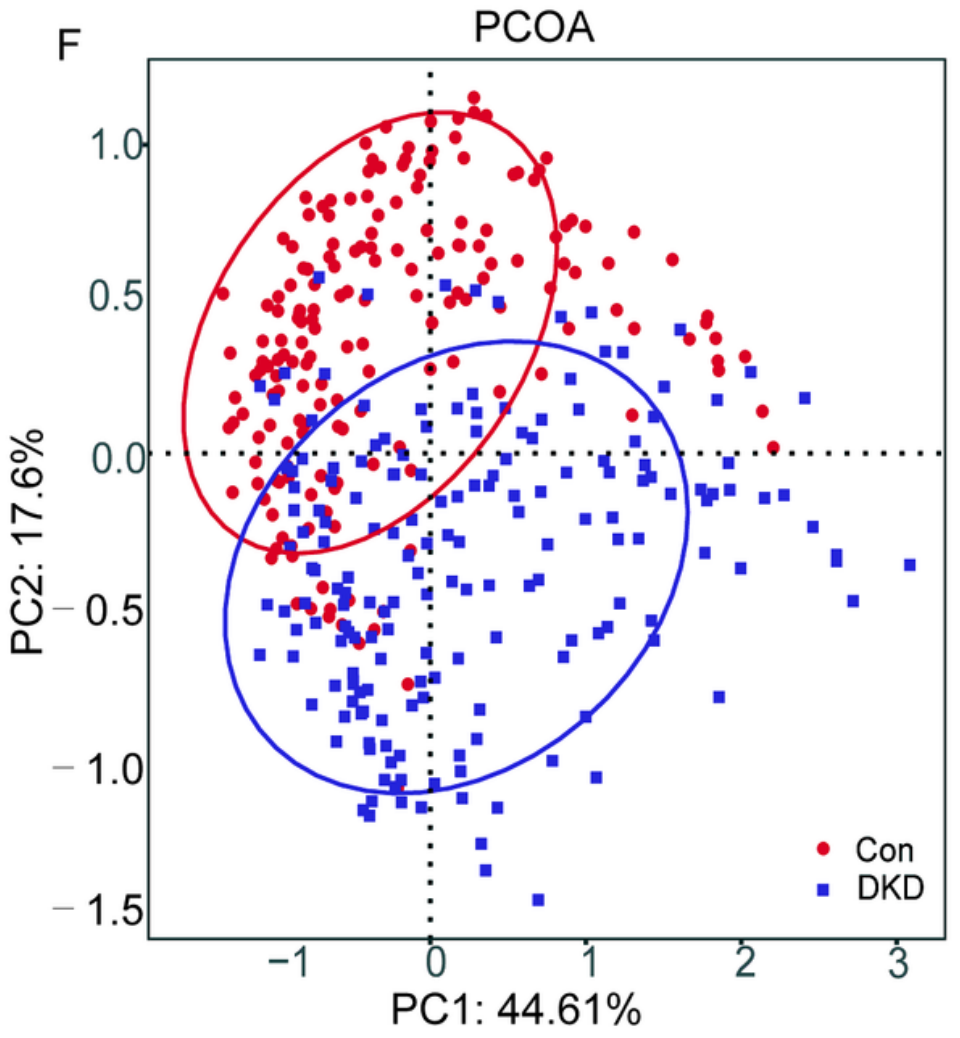
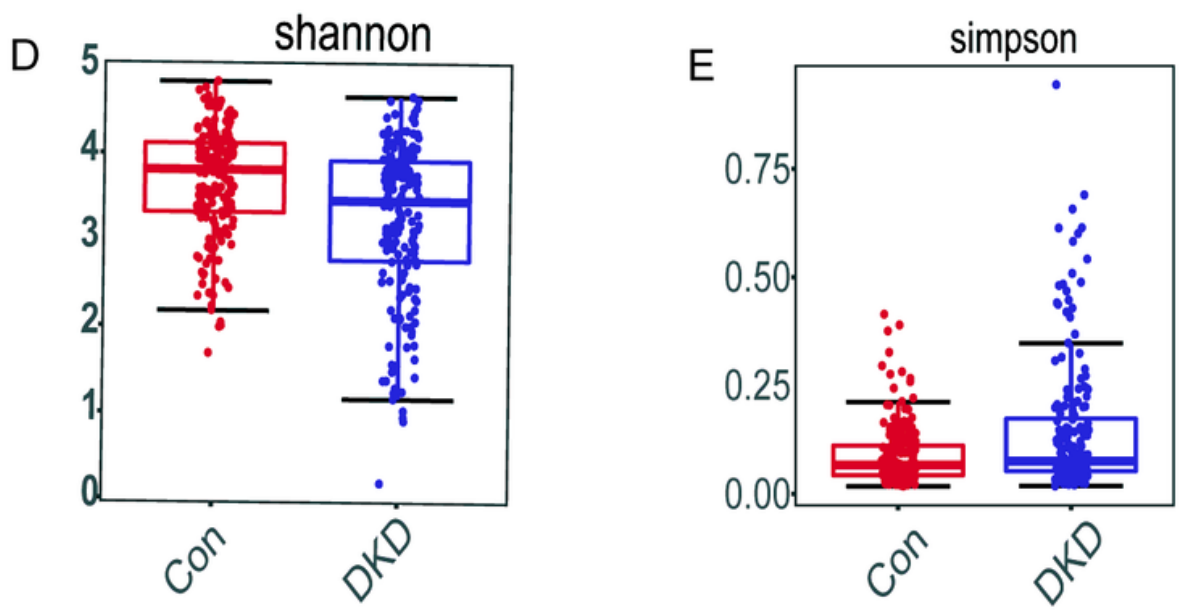

Figure 1 
Decreased bacterial diversity in DKD. (A) Venn diagram showed there were 170 unique OTUs in DKD group and 2161 shared OTUs between groups. Bacterial richness and diversity (including richness and evenness) were assessed by Ace/Chao index ( $P=1.34 \mathrm{e}-05 / 1.08 \mathrm{e}-06$; $\mathrm{B}-\mathrm{C})$ and Shannon/Simpson index $(P=5.19 e-06 / 0.0048 ; D-E)$. (F) Beta diversity visualized dissimilarity of microbial community among samples using PCoA analysis based on unweighted UniFrac algorithum (PERMANOVA test:R2 $=0.0074$; $P=0.001)$. DKD: diabetic kidney disease; Con: healthy controls; OTU: operational taxonomic unit; PCoA: principal co-ordinates analysis; PERMANOVA: permutational multivariate analysis of variance.

A

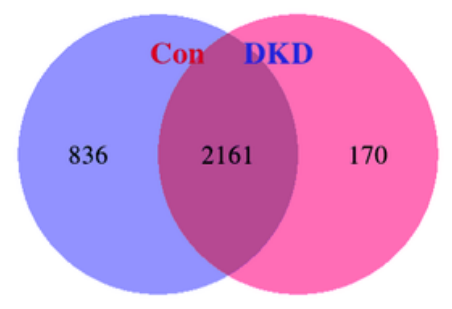

B

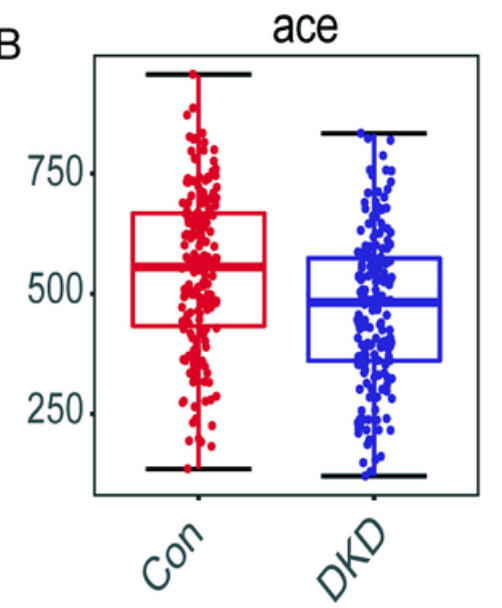

C

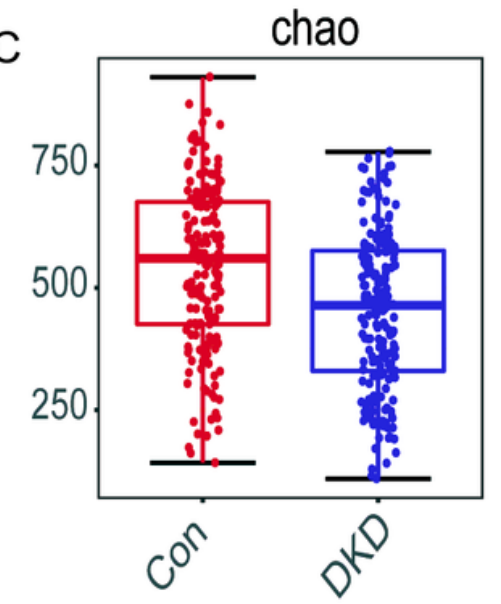

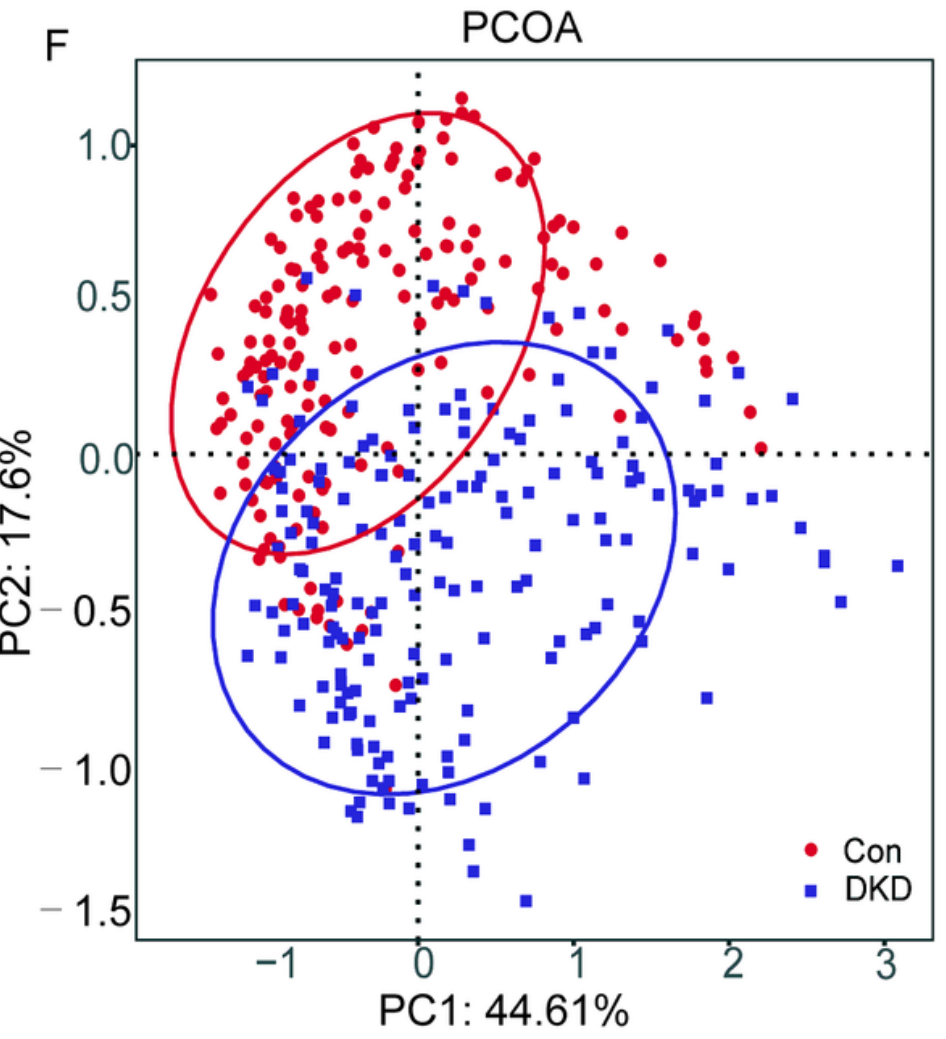
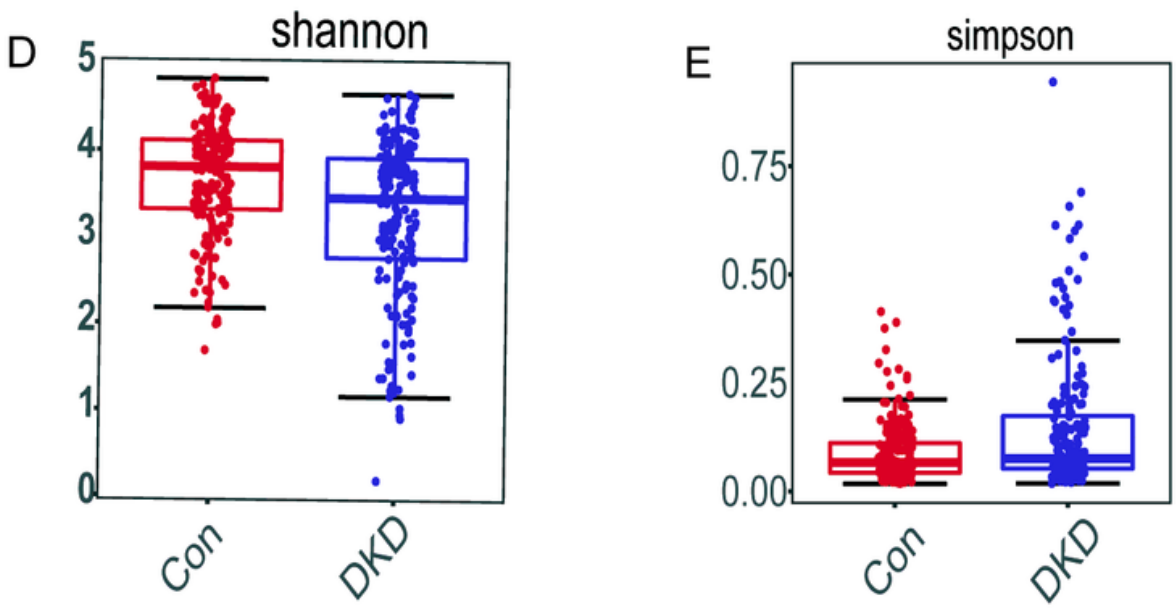

Figure 1

Decreased bacterial diversity in DKD. (A) Venn diagram showed there were 170 unique OTUs in DKD group and 2161 shared OTUs between groups. Bacterial richness and diversity (including richness and 
evenness) were assessed by Ace/Chao index ( $P=1.34 \mathrm{e}-05 / 1.08 \mathrm{e}-06$; $\mathrm{B}-\mathrm{C})$ and Shannon/Simpson index $(P=5.19 e-06 / 0.0048 ; D-E)$. (F) Beta diversity visualized dissimilarity of microbial community among samples using PCoA analysis based on unweighted UniFrac algorithum (PERMANOVA test:R2 $=0.0074$; $P=0.001)$. DKD: diabetic kidney disease; Con: healthy controls; OTU: operational taxonomic unit; PCoA: principal co-ordinates analysis; PERMANOVA: permutational multivariate analysis of variance.

A

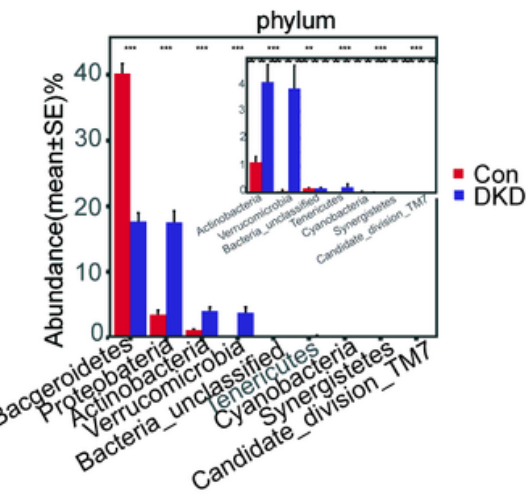

$B$

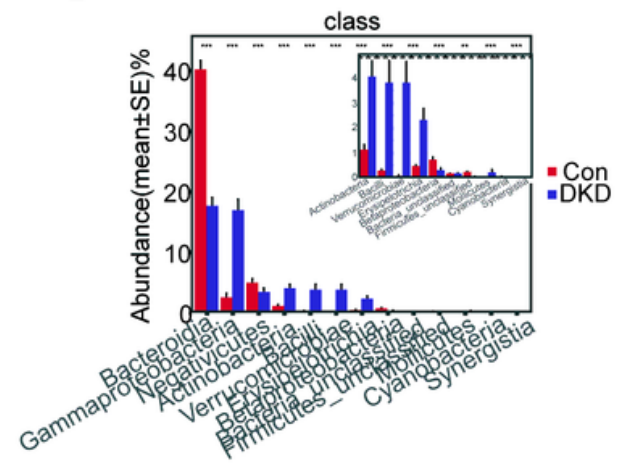

C

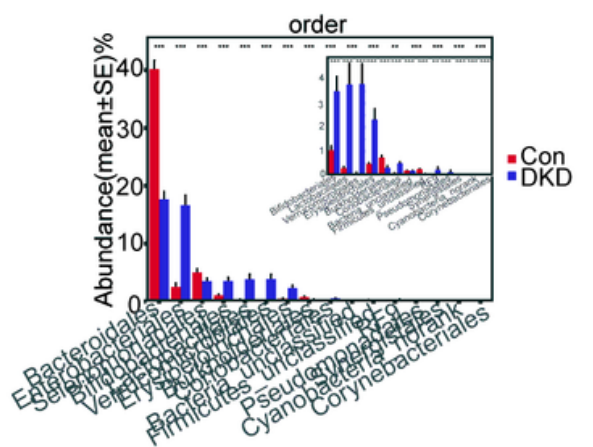

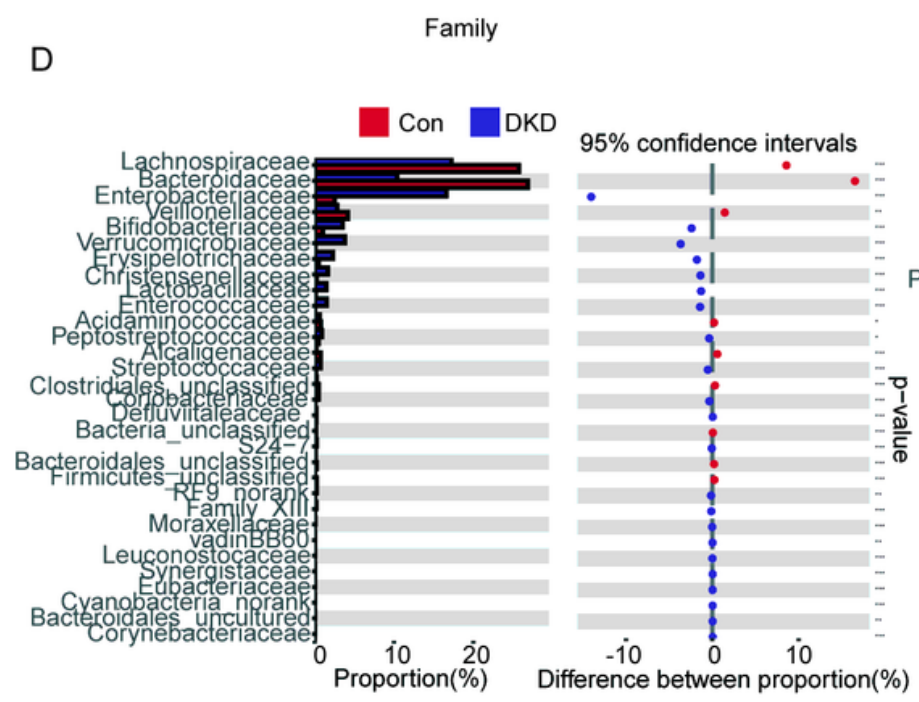

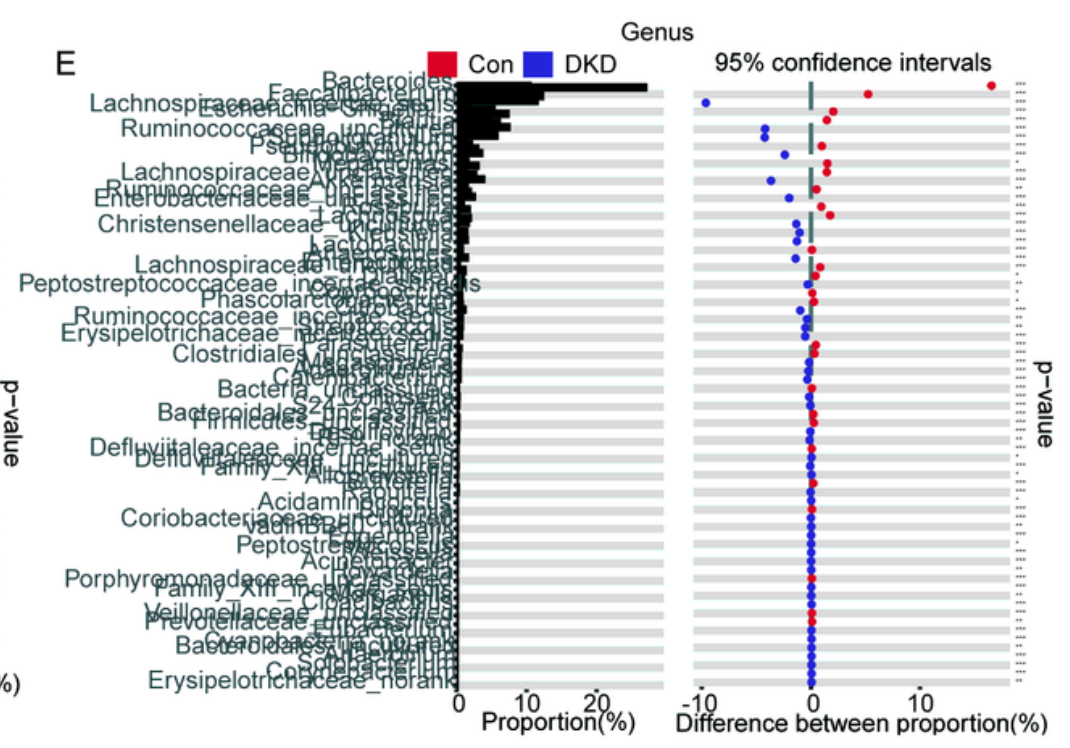

\section{Figure 2}

Comparison of microbial community between DKDs and controls. Relative abundance of 9 bacteria at the phylum level (A), 13 at the class level (B), 16 at the order level (C), 31 at the family level (D) and 70 at the genus level (E) were identified as significantly different using Wilcoxon rank-sum test. ${ }^{*} P<0.05 ; * \star P<0.01$; $\star * * \mathrm{P}<0.001$. 
A

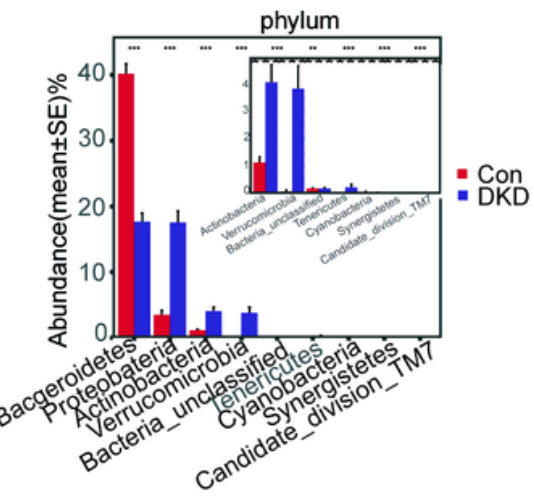

B

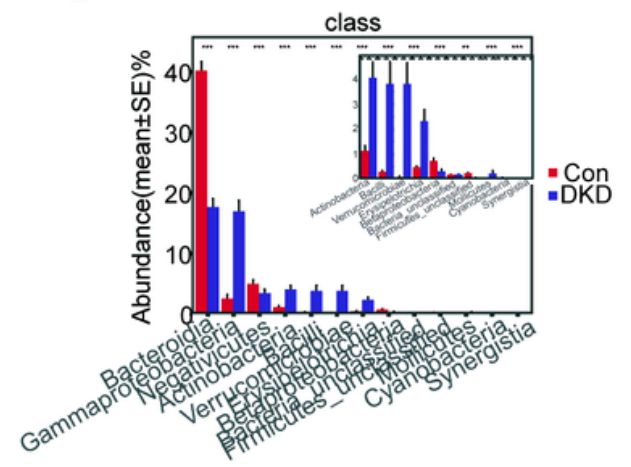

C

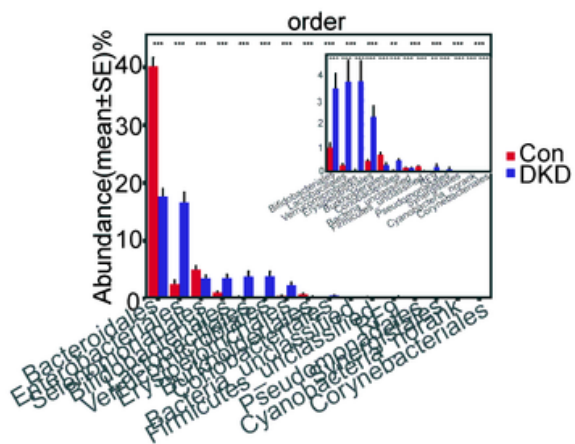

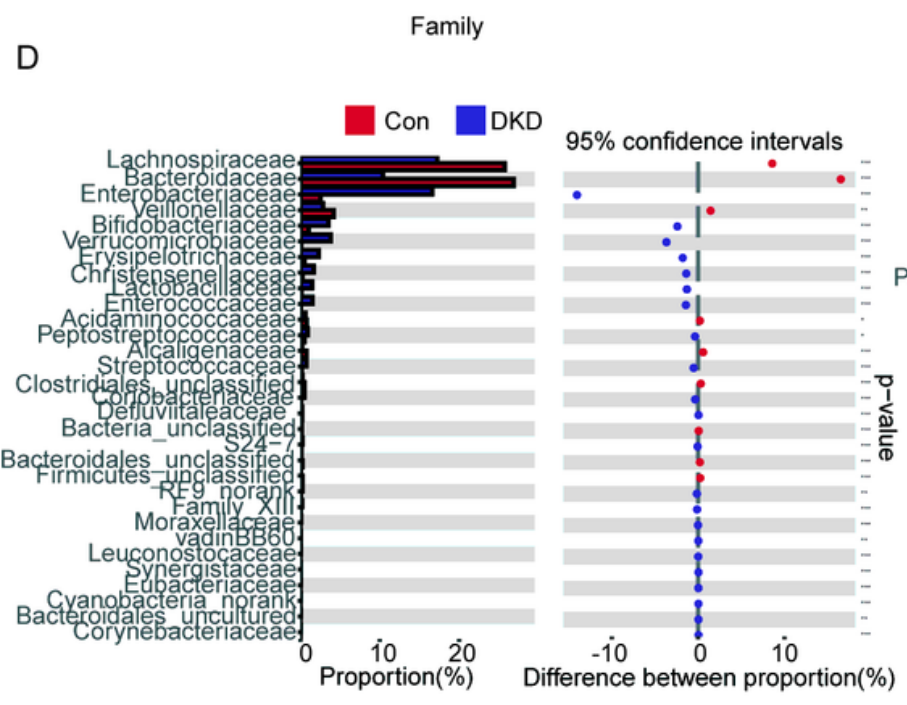

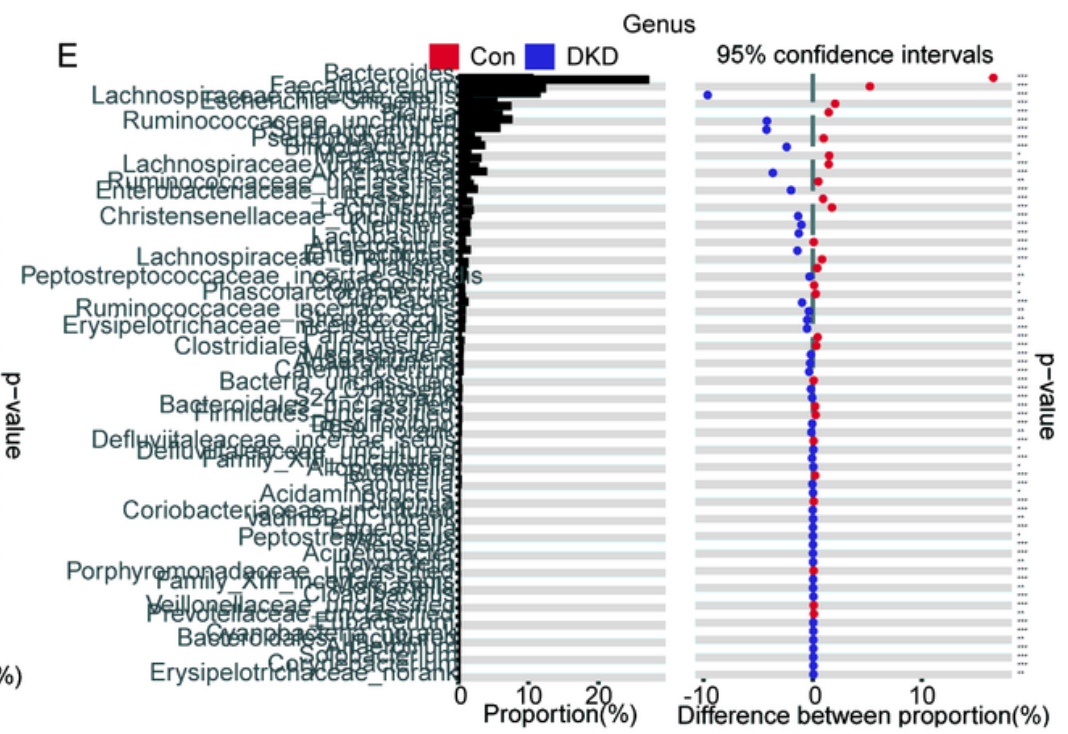

Figure 2

Comparison of microbial community between DKDs and controls. Relative abundance of 9 bacteria at the phylum level (A), 13 at the class level (B), 16 at the order level (C), 31 at the family level (D) and 70 at the genus level (E) were identified as significantly different using Wilcoxon rank-sum test. ${ }^{*} P<0.05 ; * \star P<0.01$; $\star \star \star P<0.001$.

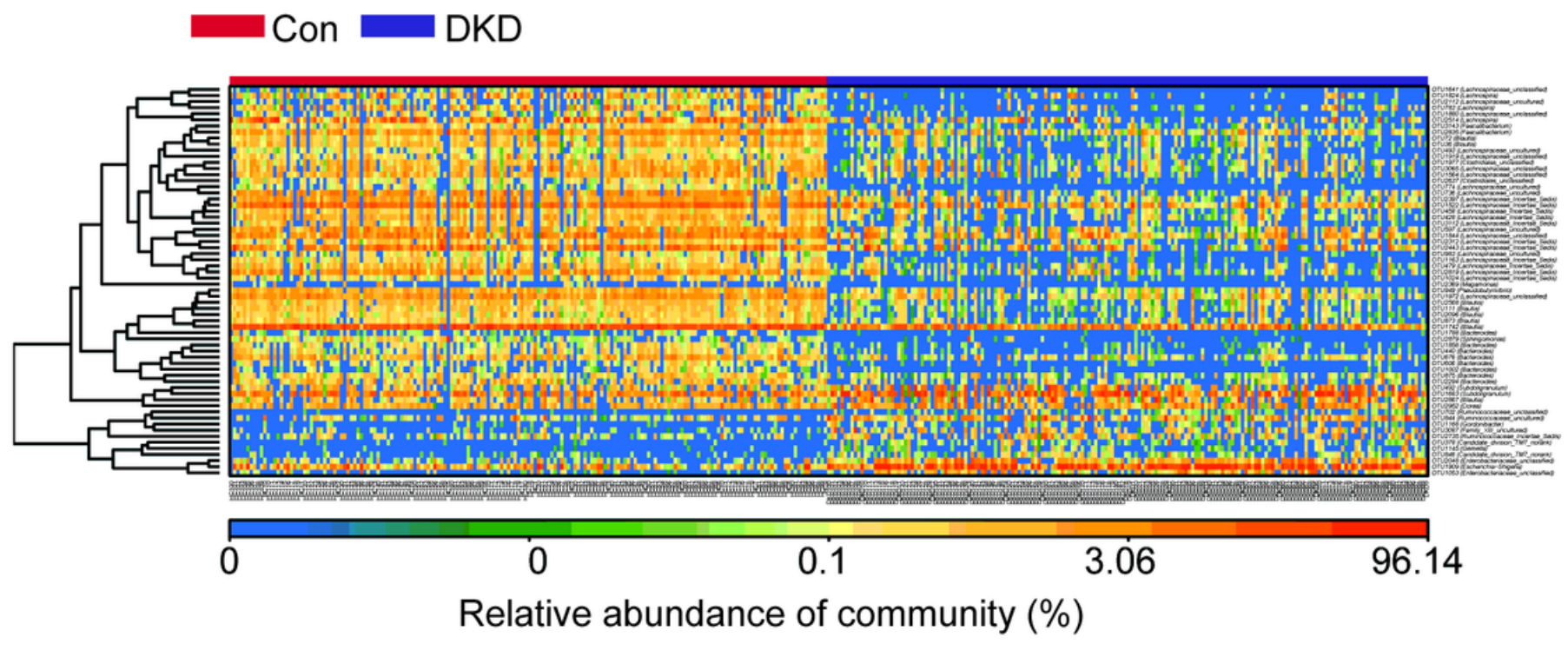

Page $15 / 23$ 


\section{Figure 3}

Distribution of key OTUs in each sample. Analysis of random forest was performed on all observed OTUs to identify potential markers. The heatmap showed that there was a significant difference between DKD group and controls based on 73 OTUs (importance value assigned by random forest larger than 0.001).

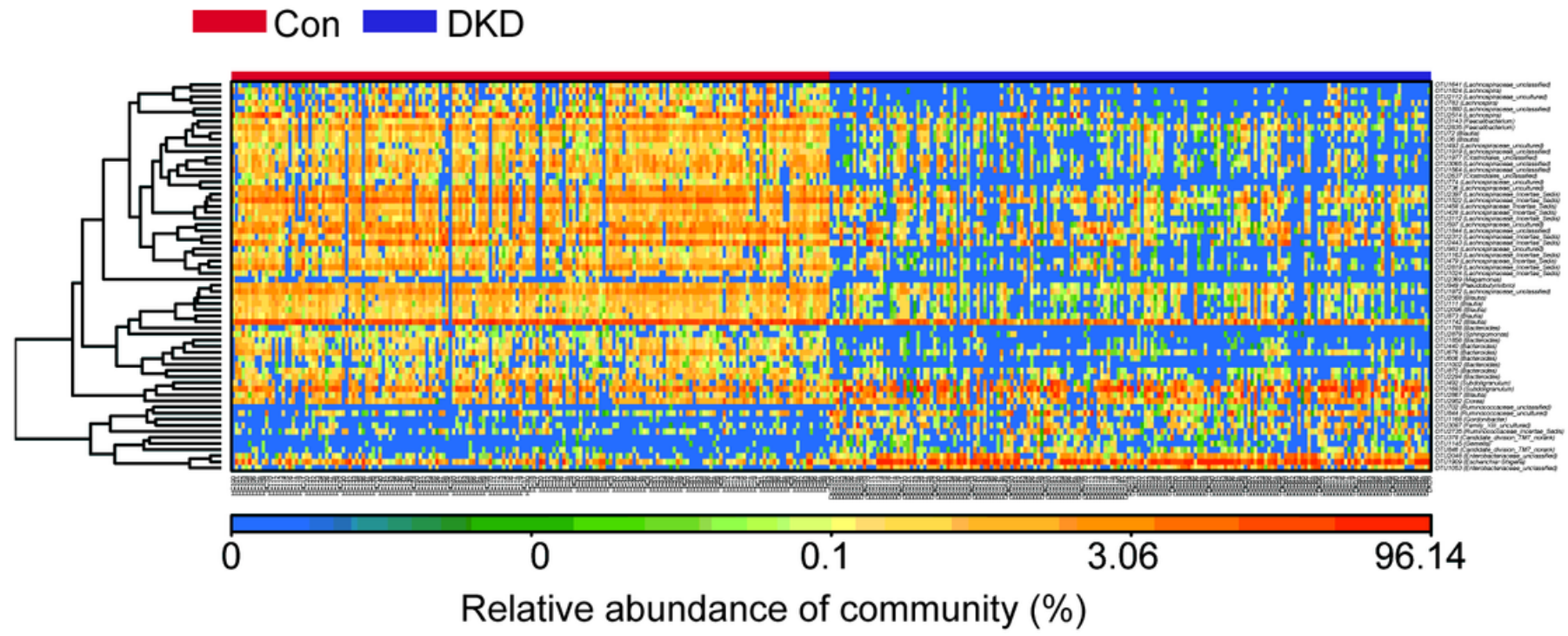

Figure 3

Distribution of key OTUs in each sample. Analysis of random forest was performed on all observed OTUs to identify potential markers. The heatmap showed that there was a significant difference between DKD group and controls based on 73 OTUs (importance value assigned by random forest larger than 0.001 ). 
A

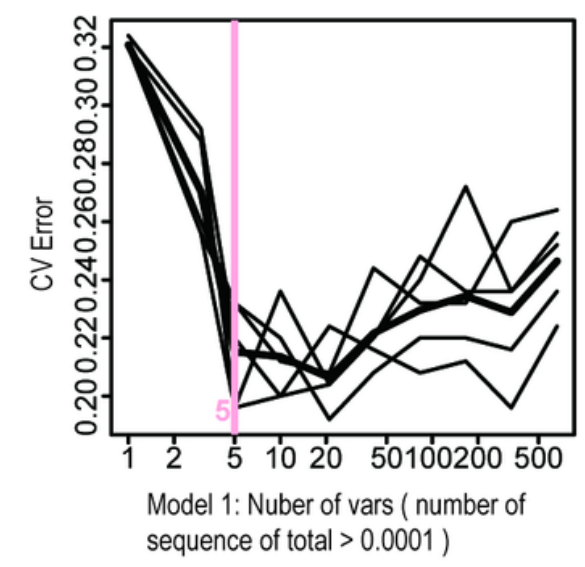

D

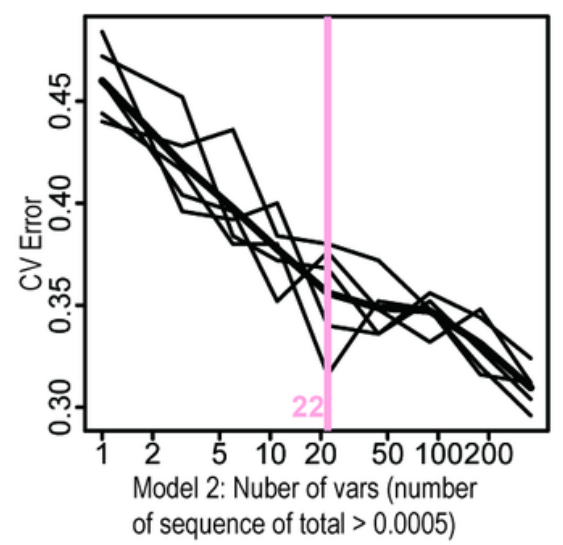

B

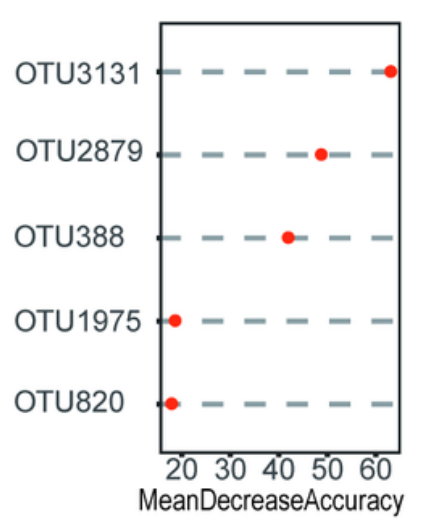

$E$

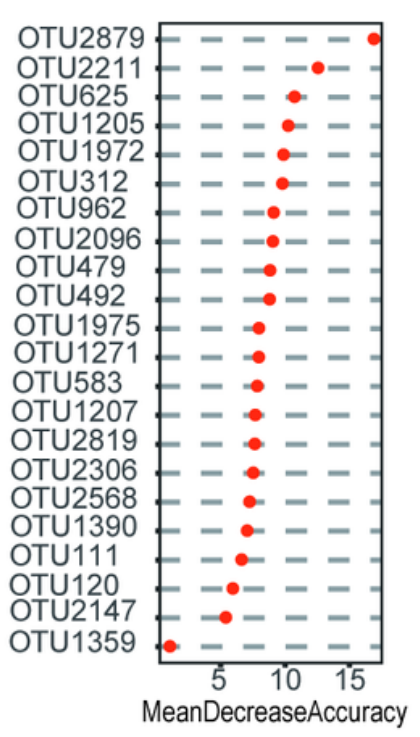

C

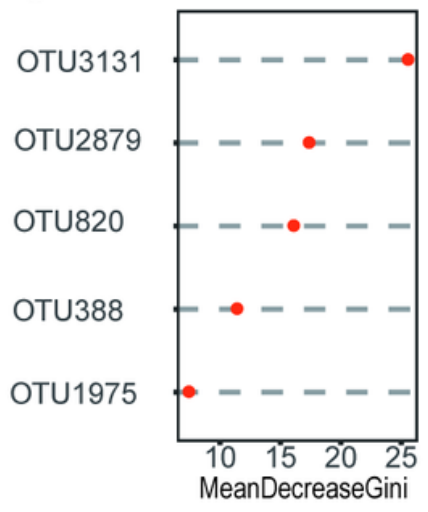

$\mathrm{F}$

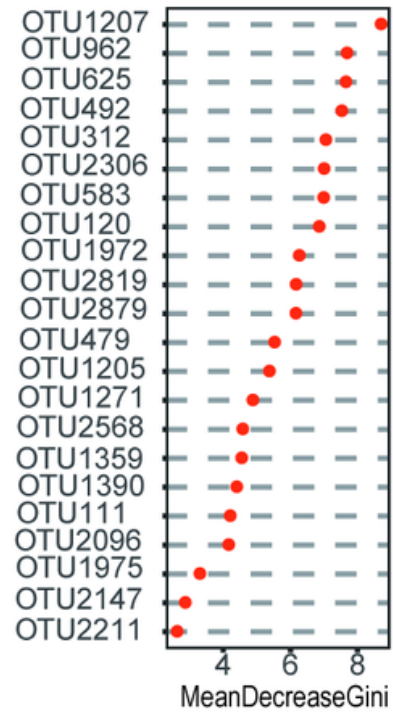

Figure 4

Characterization of the 2 diagnostic models. Model 1: (A) Five OTUs were identified as the optimal set. Relative importance (B) and stability of those OTUs in the predictive model were assessed by mean decrease of accuracy and Gini. Model 2: (B) Twenty-two OTUs were set as another diagnostic model, whose contributions and stability in classifier construction were shown in (E) and (F). 
A

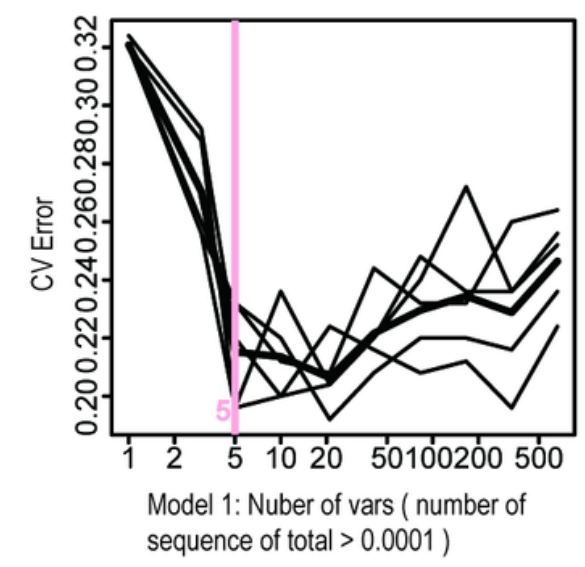

D

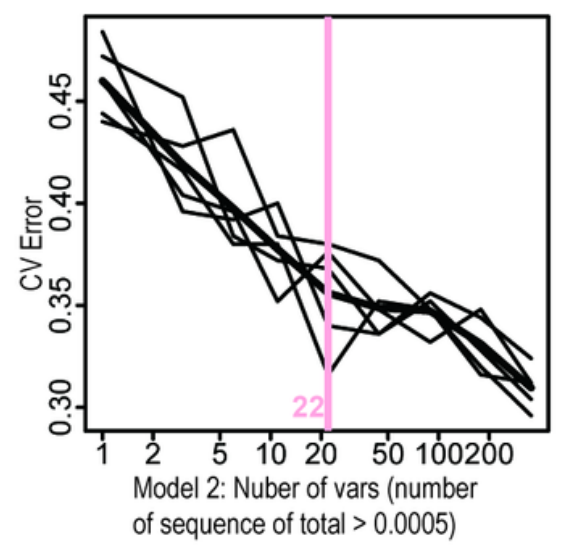

B

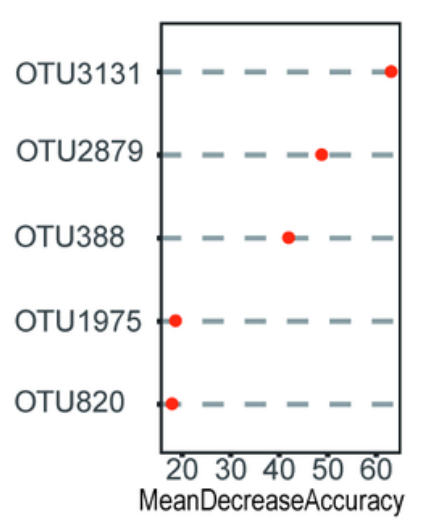

E

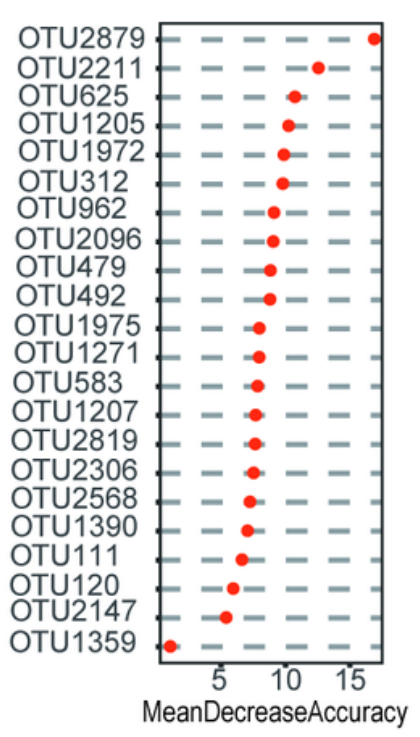

C

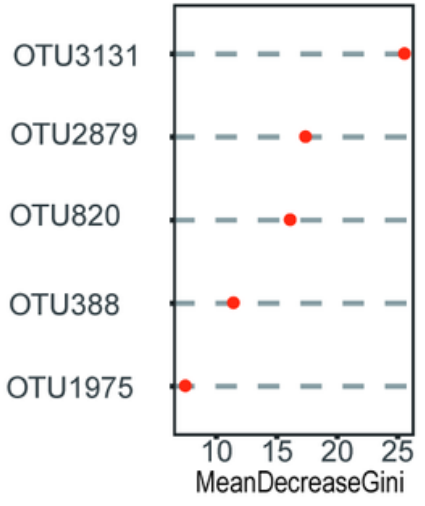

$\mathrm{F}$

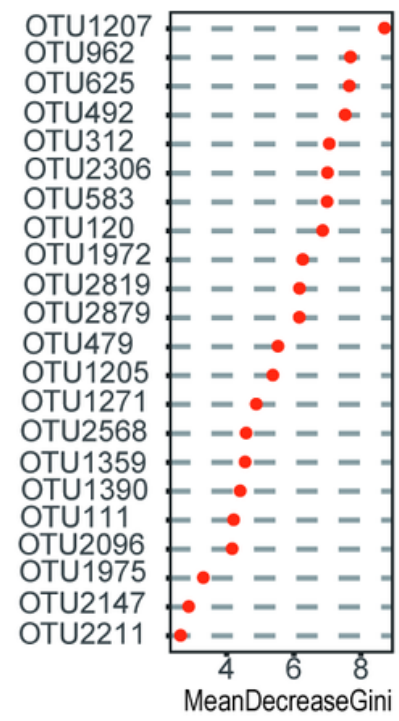

Figure 4

Characterization of the 2 diagnostic models. Model 1: (A) Five OTUs were identified as the optimal set. Relative importance (B) and stability of those OTUs in the predictive model were assessed by mean decrease of accuracy and Gini. Model 2: (B) Twenty-two OTUs were set as another diagnostic model, whose contributions and stability in classifier construction were shown in (E) and (F). 

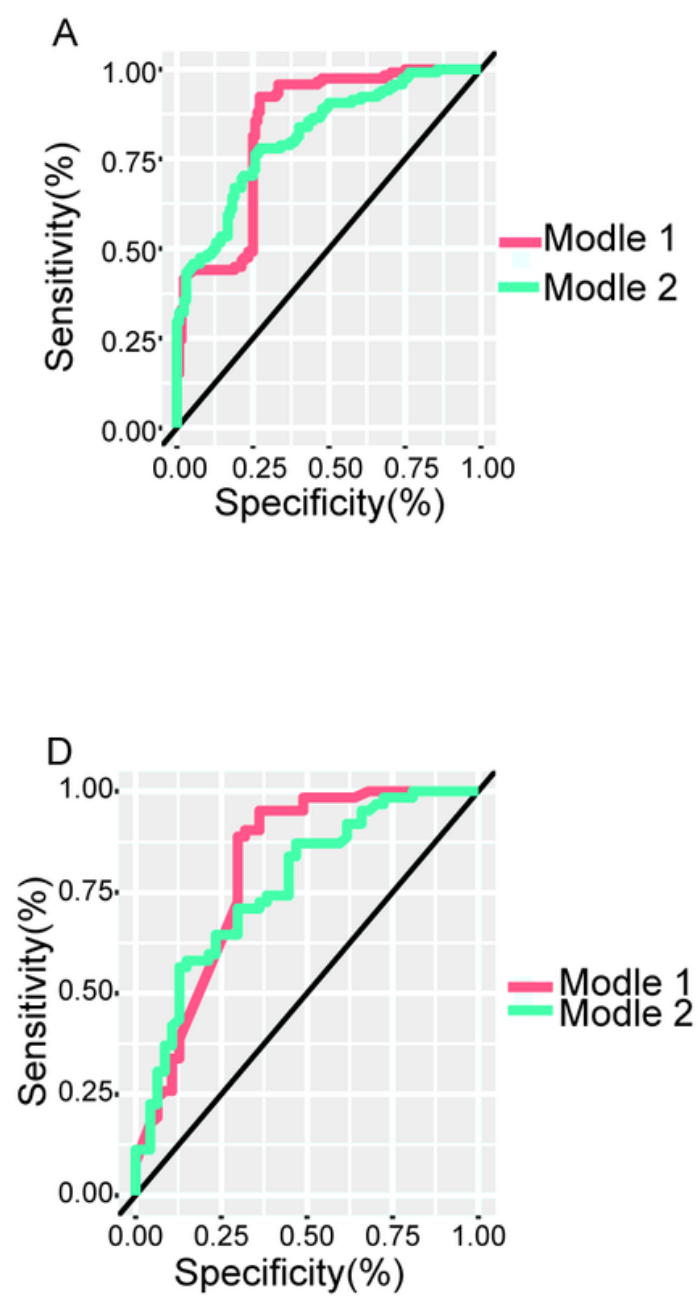

B

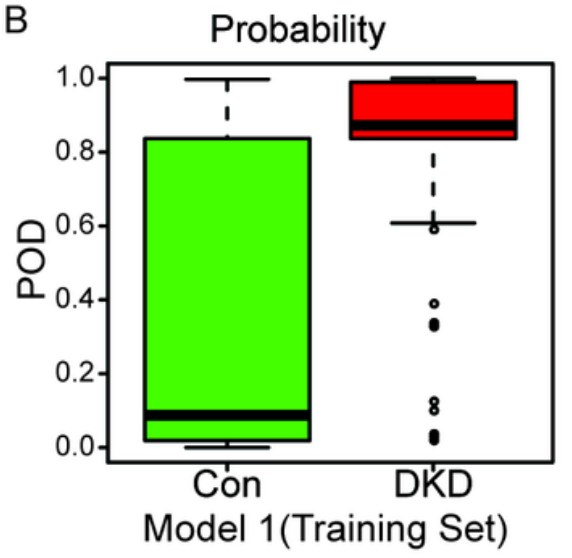

$\mathrm{E}$

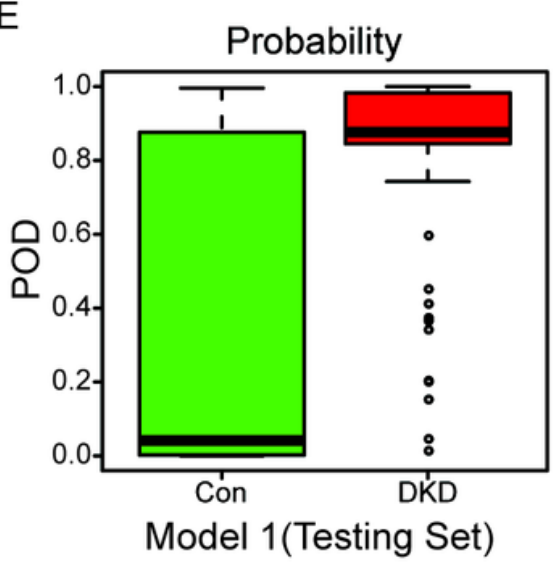

C
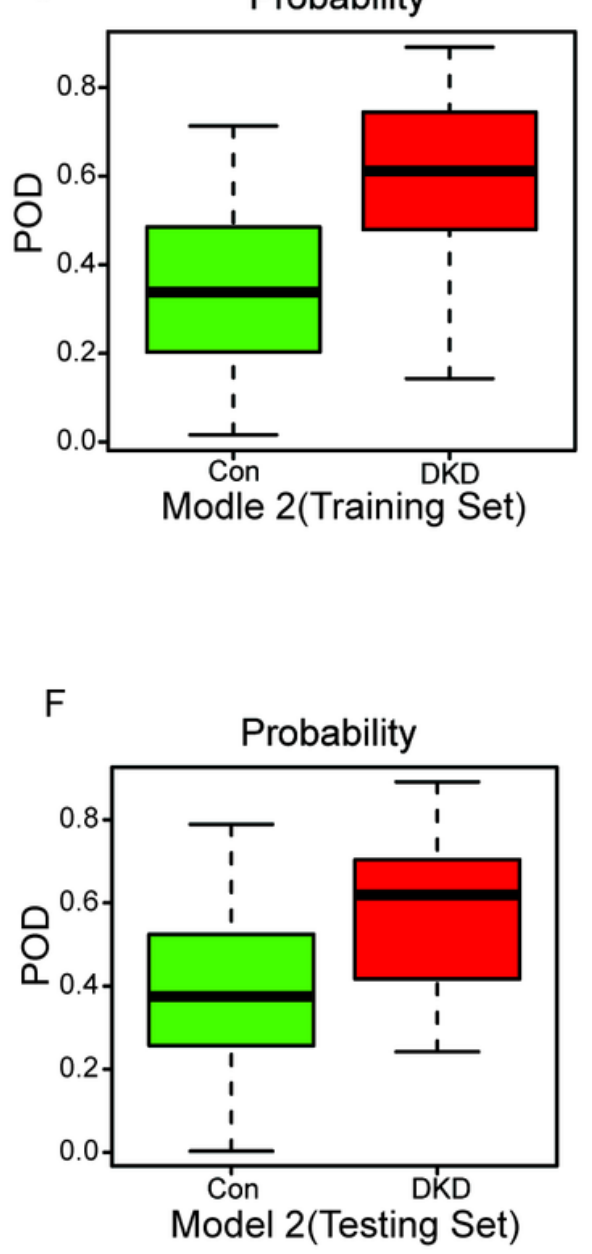

\section{Figure 5}

Predictive performance of microbial markers for DKD identification. With five trails of five-fold cross validation performed on random forest model, we identified two OTU sets to separate DKDs from healthy controls. (A) Diagnostic power of the two models in training set including 118 DKDs and 132 healthy controls were assessed by AUC. Correspondingly, increased average PODs based on model 1 (B) or model 2 (C) were observed in DKD. (D) The classifying ability of model 1 and model 2 was validated in a testing set including 62 DKDs and 47 healthy controls. The model 1- $(E)$ or 2- $(F)$ based POD value was higher in DKD group than Con group. AUC: area under the ROC curve; ROC: receiver operating characteristic curve. 

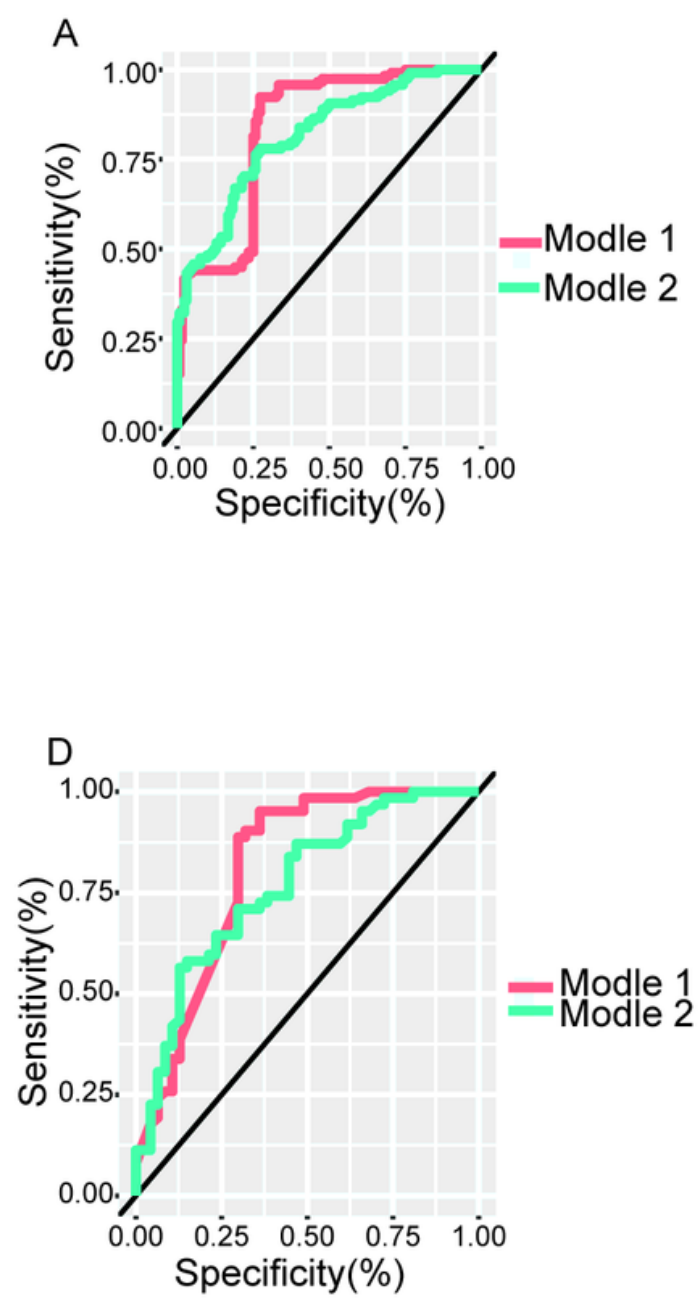

B

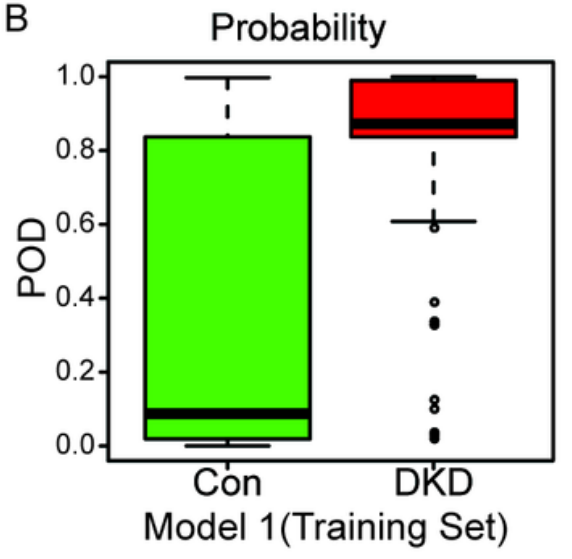

$\mathrm{E}$

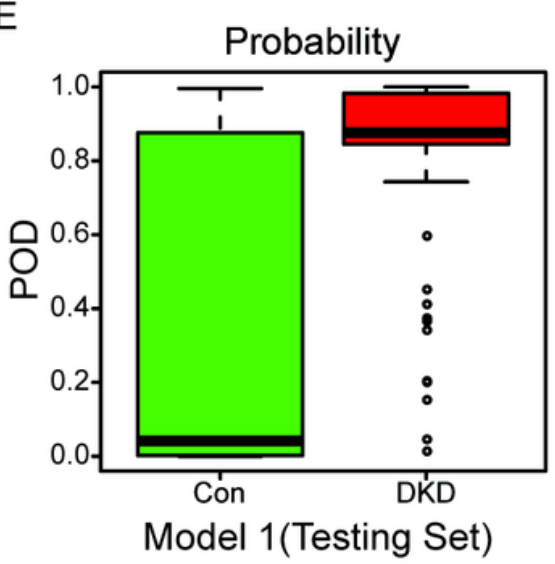

C
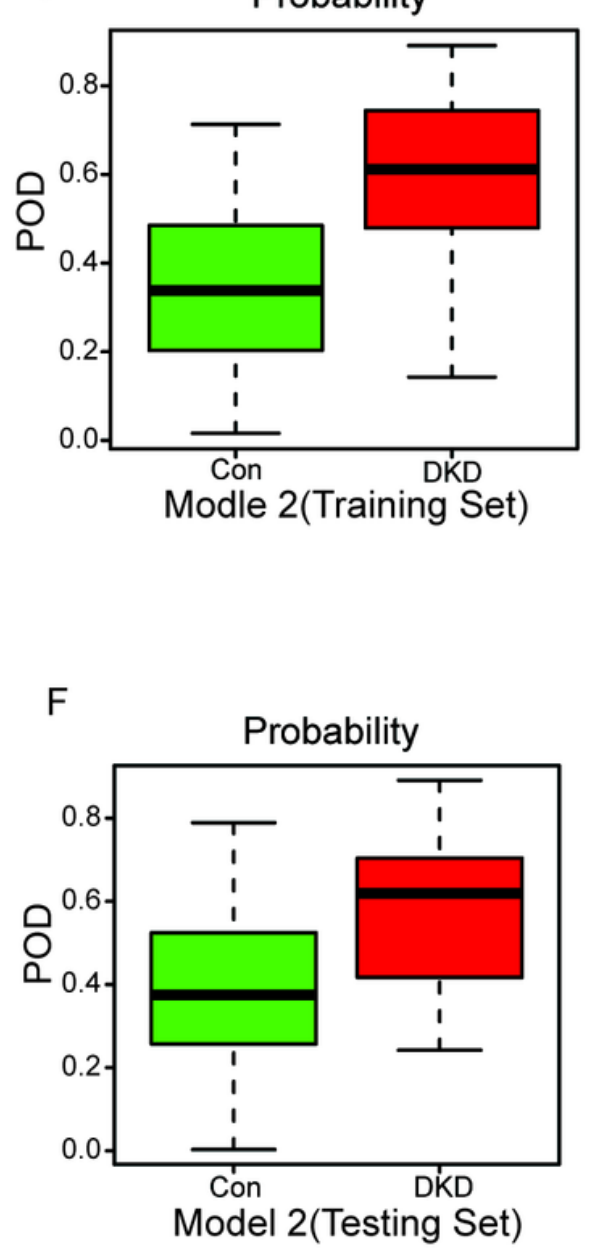

\section{Figure 5}

Predictive performance of microbial markers for DKD identification. With five trails of five-fold cross validation performed on random forest model, we identified two OTU sets to separate DKDs from healthy controls. (A) Diagnostic power of the two models in training set including 118 DKDs and 132 healthy controls were assessed by AUC. Correspondingly, increased average PODs based on model 1 (B) or model 2 (C) were observed in DKD. (D) The classifying ability of model 1 and model 2 was validated in a testing set including 62 DKDs and 47 healthy controls. The model 1- $(E)$ or 2- $(F)$ based POD value was higher in DKD group than Con group. AUC: area under the ROC curve; ROC: receiver operating characteristic curve. 
A

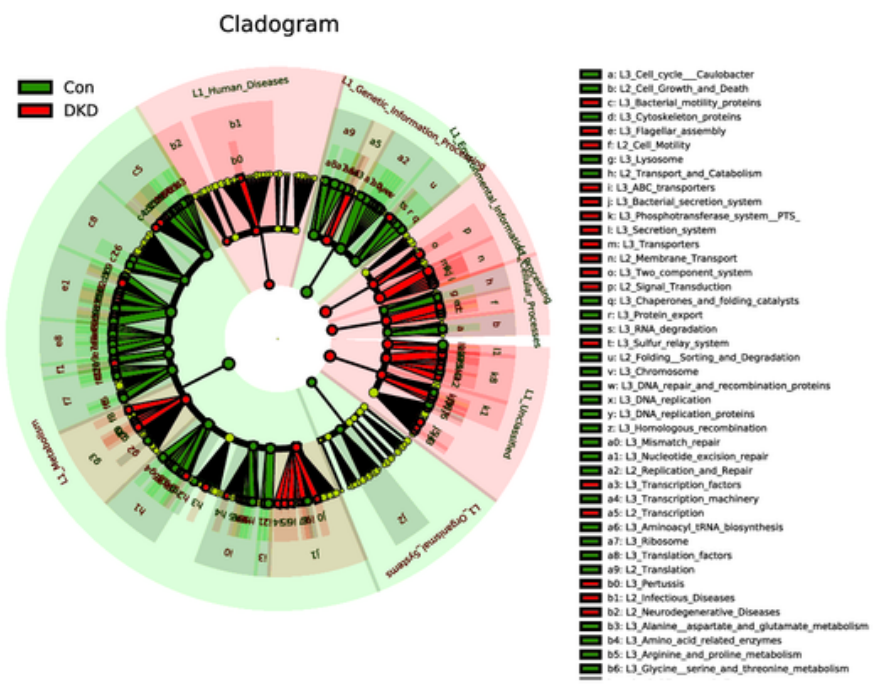

B

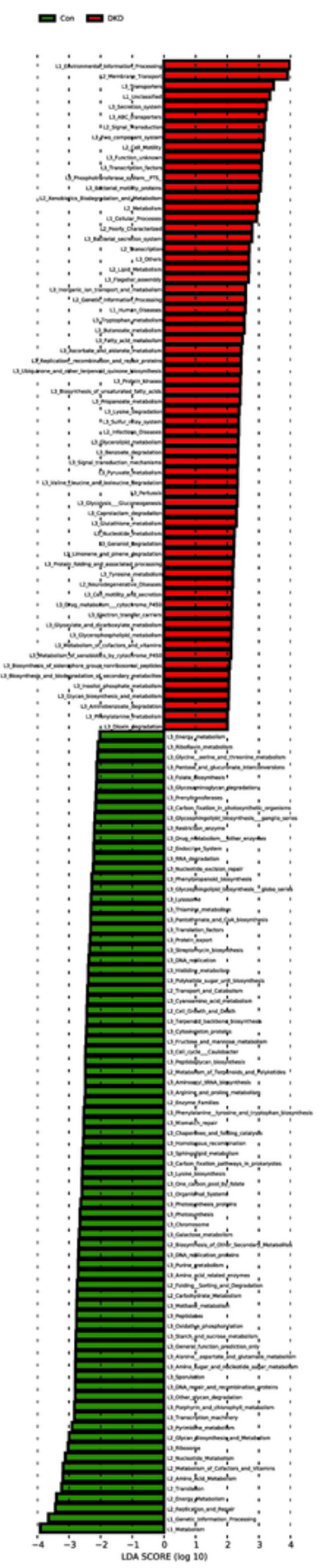

Figure 6

Microbiome-associated functional changes in both groups. Distribution (A) and comparison (B) of KEGG metabolic pathways in DKD and Con group were showed at taxonomic level using LEfSe analysis. LEfSe: linear discriminant analysis (LDA) effect size. 
A

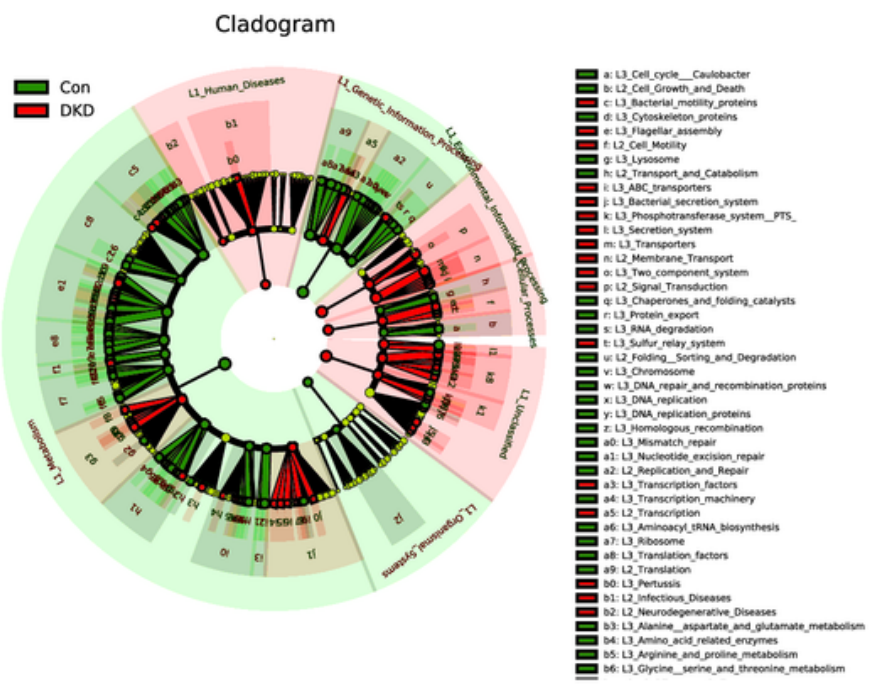

B

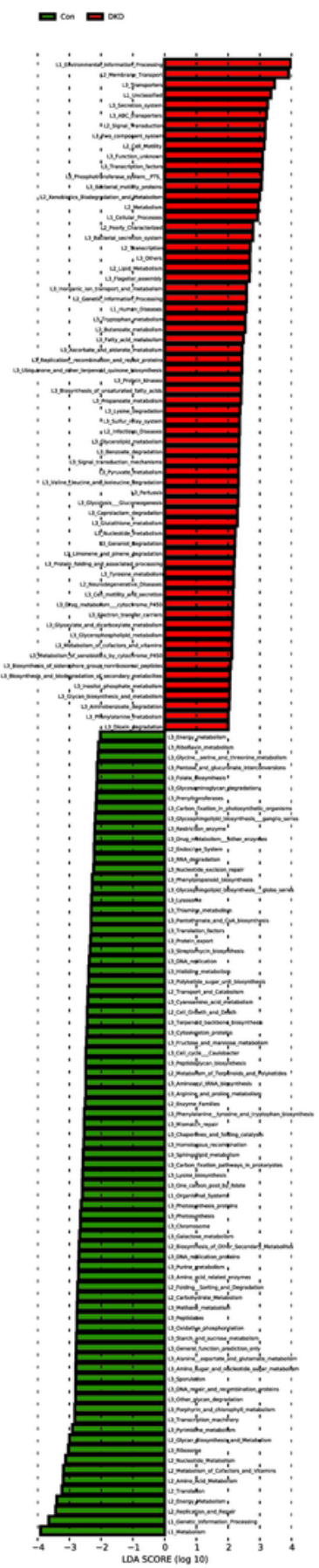

Figure 6

Microbiome-associated functional changes in both groups. Distribution (A) and comparison (B) of KEGG metabolic pathways in DKD and Con group were showed at taxonomic level using LEfSe analysis. LEfSe: linear discriminant analysis (LDA) effect size.

\section{Supplementary Files}


This is a list of supplementary files associated with this preprint. Click to download.

- FigureS1.tif

- Figures1.tif

- Figures2.tif

- Figures2.tif

- Figures3.tif

- FigureS3.tif

- FigureS4.tif

- FigureS4.tif

- FigureS5.tif

- Figures5.tif

- File1.docx

- File1.docx 